\title{
Method of visualization on droplet dynamics using two optical systems
}

\author{
Anna M. Shonina ${ }^{1,2, *}$ \\ ${ }^{1}$ Kutateladze Institute of Thermophysics SB RAS, Novosibirsk, Russia \\ ${ }^{2}$ Novosibirsk State University (NSU), Novosibirsk, Russia
}

\begin{abstract}
The methods of optical visualization of droplet dynamics are described. One of them for top view uses microscope system with CCDcamera and the second is a high-resolution shadow technique. Droplet evaporation dynamics are recorded and processed using special programs. Evolution of droplet base diameters and volumes are presented.
\end{abstract}

\section{Introduction}

The wettability of a surface is usually described by the contact angle, measured within the liquid at the three phase contact line. Due to the topographical peculiarities of the solid surfaces, in reality the contact angle does not exhibit a unique value. The contact angle hysteresis, which is the difference between advancing and receding contact angles, plays an important role on wetting and dewetting processes. The nanostructured surfaces allow control and significantly influence on the processes of heat transfer $[1,2]$ and hydrodynamics $[3,4]$ in a variety of applications. The roughness and the number of microholes can be increased. On the other hand, contact angle, capillary effects, porosity can be controlled. This leads to change in the nature of two-phase flow, hydraulic resistance, critical heat flux, condensation, evaporation, etc. One of the most actual issues is the behavior of the three phase contact line $[5,6]$ on surfaces with different coatings $[7,8]$. The methods of optical visualizations in order to have high-resolution system is challenging issue $[9,10]$.

This paper presents the results of applications of two optical methods for three phase contact line dynamics during evaporation of hanging droplet.

\section{Visualization systems}

Scheme of experimental setup is presented in Fig. 1. The glass slides (Struers) are used as substrate. The contact angle of water droplet on glass slides has been measured using KRUSS DSA-100 (Fig. 2). And it is found to be of 16 degrees. The ultrapure water (MilliQ) is used as the working fluid. The liquid of volume of $2 \mu 1$ is deposited to the glass surface using a precision syringe pump Cole Parmer. The syringe of $0.5 \mathrm{ml}$ volume is used in order to make a droplet of the precise volume. An atmosphere is controlled by Testo

*Corresponding author: annamishonina@gmail.com 
device and barometer. Top view visualization of evaporation dynamics has been done using Olympus BX51 microscope with different lenses and monochrome camera Point Grey. The resolution of $1 \mu \mathrm{m}$ per pixel is provided by using $10 \times$ lens and $100 \mathrm{~nm}$ per pixel by $100 \times$ lens. Recording frequency is from 10 to $40 \mathrm{fps}$. Side view of droplet profile is recorded using the shadow method (Fig. 1) providing resolution of $3 \mu \mathrm{m}$ per pixel by the present time. Contact angles are measured at different times using Advance software of KRUSS DSA-100. Dynamics of the three phase contact line during droplet evaporation on the clean glass are studied.

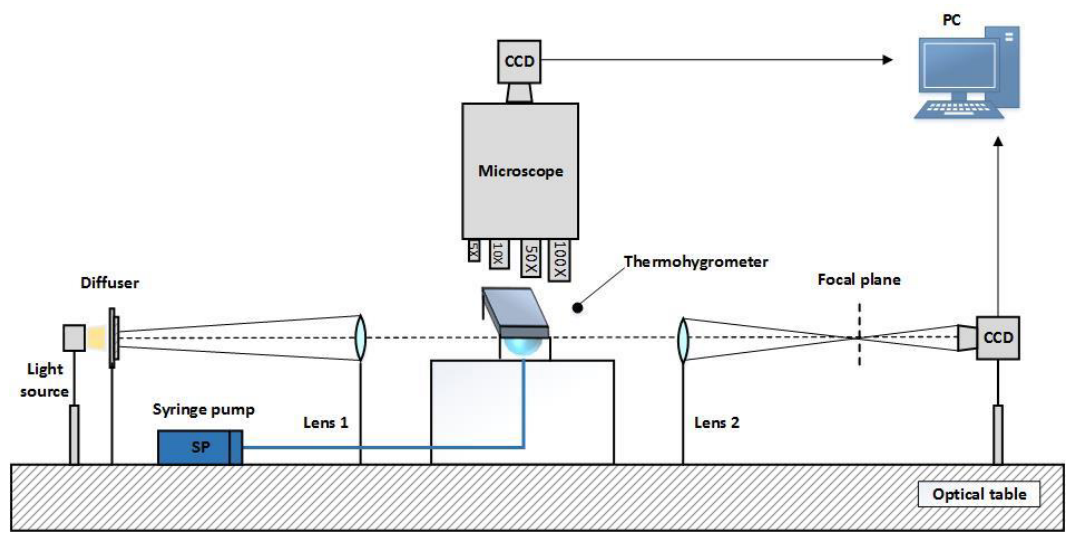

Fig. 1. Scheme of experimental setup.

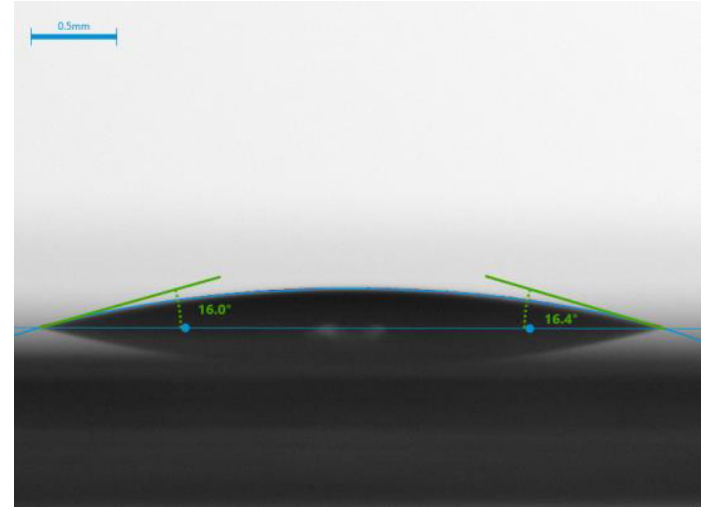

Fig. 2. Contact angle measurement by KRUSS DSA-100.

\section{Results and discussions}

The examples of images taken by two techniques are presented in Fig. 3. Finally, records are processed in ImageJ and Advance (KRUSS) software. The data on time, diameter, area and perimeter of droplet base, volume, velocity and contact angle are obtained using these programs. Some characteristic plots of evolutions of droplet volume via time (obtained from the side view) and of droplet diameter (obtained from the top view) are shown in Fig. 4a and Fig. 4b, respectively. Here, zero time is the time of complete droplet disappearance. 


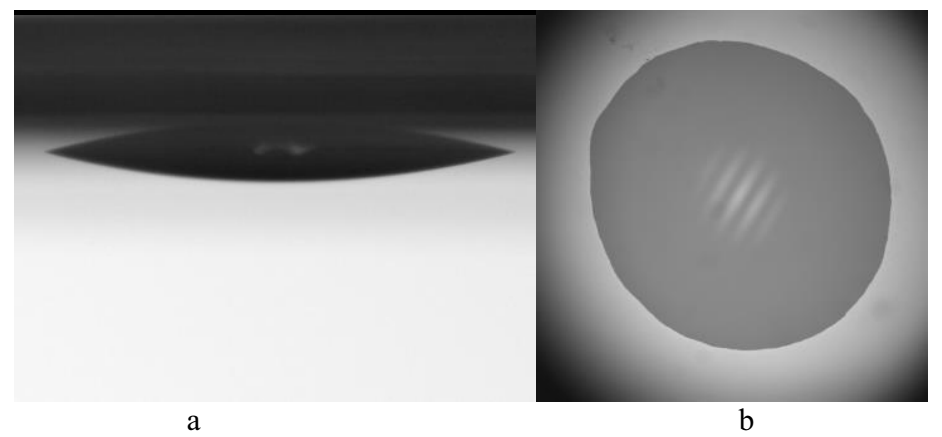

Fig 3. Images of droplet on the glass substrate. Initial volume is $2 \mu 1 . a$-side view by shadow method, $b$-top view by using microscope.
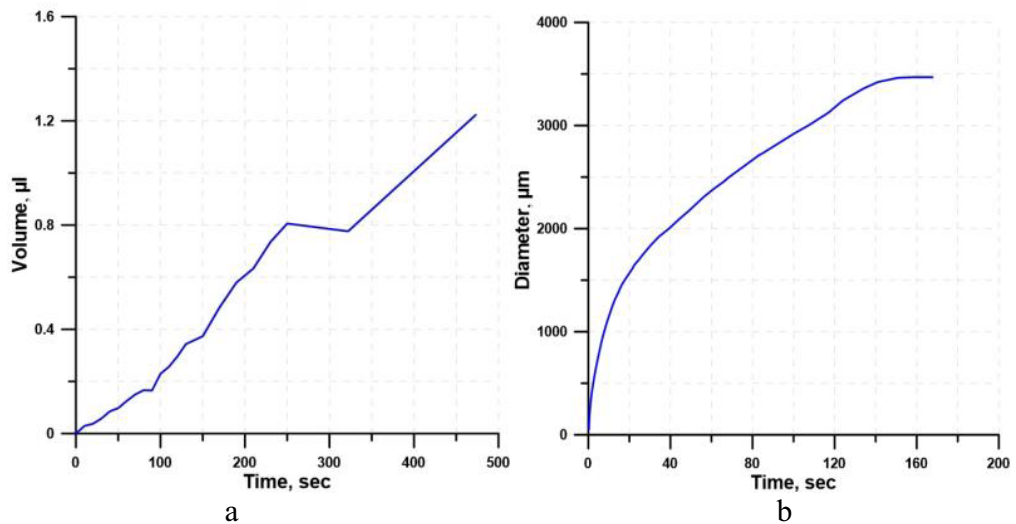

$\mathrm{b}$

Fig. 4. Volume and diameter plots. $a$-side view by shadow method, $b$-top view by using microscope.

\section{Conclusion}

In conclusion, two optical methods for visualization of droplet dynamics have been adopted. One of them for the top view uses microscope system with CCD-camera and the second is a high-resolution shadow technique. The top view technique can provide with the resolution of up to $100 \mathrm{~nm}$ per pixel. The synchronization of top and side video registrations via time is organized.

The authors gratefully acknowledge the support of this work by the Ministry of Education and Science of the Russian Federation (Agreement 14.616.21.0016, project identifier RFMEFI61614X0016).

\section{References}

1. N. Miljkovic, R. Enright, Y. Nam, K. Lopez, N. Dou, J. Sack, E.N. Wang, Nano Lett. 13, 179 (2013)

2. C. Lin, Droplet Impact and Evaporation on Nanotextured Surface for High Efficient Spray Cooling in An Overview of Heat Transfer Phenomena ed. by S. N. Kazi (In Tech, Croatia, 2012)

3. E.Y. Gatapova, V.S. Ajaev, O.A. Kabov, JETP Letters 101, 160 (2015) 
4. V.S. Ajaev, E.Y. Gatapova, Kabov O.A., Adv. Colloid Interfac. 228, 92 (2016)

5. A. Zou, A. Chanana, A. Agrawal, P.C. Wayner Jr., S.C. Maroo, Sci. Rep. 6, 20240 (2016)

6. E.Ya. Gatapova, O.A. Kabov, Microgravity Sci. Tec. 19, 132 (2007)

7. A. Dubov, A. Mourran, M. Moeller, O. Vinogradova, J. Chem. Phys. 141, 074710 (2014)

8. E.Ya. Gatapova, A.A. Semenov, D.V. Zaitsev, O.A. Kabov, Colloid Surf. A 441, 776 (2014)

9. S.S. Panchamgam, A. Chatterjee, J.L. Plawsky, P.C. Wayner, Jr., Int. J. Heat Mass Tran. 51, 5368 (2008)

10. M.S. Hanchak, M.D. Vangsness, L.W. Byrd, J. S. Ervin, J.G. Jones, Appl. Phys. Lett. 103, (2013) 